\title{
Factibilidad en la elaboración de calzado de cuero para caballero: Caso de estudio
}

Feasibility in the manufacture of leather footwear for men: Case study

Juan Ennis Espinoza González ${ }^{1}$, Kléber Antonio Luna Altamirano², Marcos Eduardo Cantos Ochoa $^{3}$, Ximena Rocío González Astudillo ${ }^{4}$, Verónica Paola Chamba Esparza ${ }^{5}$.

Recibido: 10-04-2019 / Revisado: 25-04-2019 /Aceptado: 05-05-2019/ Publicado: 16-05-2019

\begin{abstract}
DOI: https://doi.org/10.33262/cienciadigital.v3i2.3.567

The objective of this investigation is to determine the feasibility of producing and marketing leather footwear and men's soles in the city of Azogues-Ecuador, whose problem is reflected in the lack of this type of product on the market, due to prices not accessible to consumers in similar or substitute products, moreover the craftsmen who made this footwear have withdrawn from the profession and others have died. To deliver to the citizenship a product of quality elaborated in leather and sole of artisan form, represents elegance and distinction at the moment of dressing, the niche of market is centered in clients like professionals in different areas, like lawyers, academics, teachers, authorities and those people whose activities are of institutional nature. The results have demonstrated the feasibility of this undertaking as the economic profitability indicators are highly reliable and have responded with percentages according to the market, thus recommending the implementation and operation of this site.
\end{abstract}

Keywords: Feasibility, leather footwear, economic indicators.

\footnotetext{
${ }^{1}$ Universidad Católica de Cuenca, Maestrante en Administración de Empresas, Cuenca, Ecuador.jespinozag@psg.ucacue.edu.ec

${ }^{2}$ Universidad Católica de Cuenca, Unidad Académica de Administración, Cuenca, Ecuador. klunaa@ucacue.edu.ec

${ }^{3}$ Universidad Católica de Cuenca, Unidad Académica de Administración, Cuenca, Ecuador. mecantoso@ucacue.edu.ec

${ }^{4}$ Magister en Administración de Empresas, xgonzalezastudillo@gmail.com

${ }^{5}$ Universidad Católica de Cuenca, Maestrante en Contabilidad y Auditoría con Mención Tributaria, Cuenca, Ecuador. vpchambae279@psg.ucacue.edu.ec
} 


\section{Resumen}

La presente investigación tiene como objetivo determinar la factibilidad de producir y comercializar calzado de cuero y suela para caballero en la ciudad de AzoguesEcuador, cuyo problema se traduce a la poca existencia de este tipo de producto en el mercado, debido a precios no accesibles por los consumidores en productos similares o sustitutivos, a más de ello los artesanos que elaboraban este calzado se han retirado de la profesión y otros han fallecido. Entregar a la ciudadanía un producto de calidad elaborado en cuero y suela de forma artesanal, representa elegancia y distinción al momento de vestir, el nicho de mercado está centrado en clientes como profesionales en diferentes áreas, como abogados, académicos, docentes, autoridades y aquellas personas cuyas actividades son de índole institucional. Los resultados han demostrado la factibilidad de este emprendimiento ya que los indicadores de rentabilidad económicos son altamente confiables y han respondido con porcentajes acordes al mercado, con ello se recomienda la ejecución y operatividad de este emplazamiento.

Palabras clave: Factibilidad, calzado de cuero, indicadores económicos.

\section{Introducción}

En la actualidad se ha ido perdiendo el arte de confeccionar calzado de cuero artesanal, donde predominaba la habilidad, creatividad y destreza de los artesanos para la fabricación de este producto, utilizando procesos manuales. El avance tecnológico e industrial, ha propiciado que se elaboren calzado con materiales que remplacen al cuero, su estructura de manera rápida, que involucran costos bajos dando como resultado un producto de poca calidad y durabilidad. Por ello esta investigación tiene como objetivo determinar la factibilidad de producir y comercializar calzado de cuero y suela para la sociedad Azogueña, ya que en la actualidad existe carencia de este producto, traduciéndose en un problema para quienes desean vestir una prenda de calidad, la falta de artesanos, los precios altos de productos similares hacen que este calzado no llegue al nicho de mercado establecido.

Con el propósito de demostrar la factibilidad del emplazamiento, se conocerán los ingresos, costos y los flujos netos de caja proyectados que generará la fabricación de este tipo de producto, se considera la capacidad instalada para la producción. Los montos de inversión requeridos para la fabricación de calzado de cuero y suela para caballero, son relativamente bajos en comparación con la gran industria, misma que facilita tomar decisiones, pudiendo recurrir al mercado de maquinaria usada (Castillo y Cortellese, 1988). 
En los actuales momentos, el saber competir se convierte en un desafío para los nuevos emprendedores en un mercado que solicita un producto que cumpla con las expectativas de los clientes. Rosales (2000) explica que la rapidez para descubrir las nuevas tendencias en la moda, la conducta y necesidades de los potenciales clientes, los nuevos diseños, producir y comercializar los productos para las nuevas necesidades, se está cristalizando en el modelo gerencial para las empresas líderes. Por su parte García (1999), las pequeñas y medianas empresas están direccionadas a nichos de mercado específicos elaborando productos novedosos para mercados locales y nacionales, poseen mejor adaptación a los cambios de tecnologías con gran flexibilidad para satisfacer a una gran cantidad de clientes.

La factibilidad de producir y comercializar este tipo de calzado, está dado por sus indicadores clásicos de rentabilidad económica, Tasa Interna de rendimiento (TIR) y Valor actual Neto (VAN), estos fueron aceptables de acuerdo a las realidades del mercado local; este producto cuenta con materia prima de calidad, mano de obra calificada, el cual cubrirá las necesidades de los clientes generando valor agregado al consumidor. El presente estudio, está enfocado a generar desarrollo dentro de la ciudad de Azogues, mediante la introducción de un producto que cumpla con los deseos y expectativas de quienes lo soliciten.

\section{Estado del arte}

Fabricar calzado artesanal se convierte en un nuevo desafío para el mercado actual, por ello algunas investigaciones apuntan a la fabricación de calzado desde diferentes perspectivas, entre ellas se tiene: Villegas y Zapata (2007) describen el nivel de competitividad internacional, del sector del cuero y del calzado colombiano, mediante la aplicación de indicadores que, a partir de la información estadística de las variables de comercio exterior permiten establecer ciertas relaciones que dan cuenta de la importancia del producto en los mercados de los países de la Comunidad Andina de Naciones (CAN). Mosquera, Rojas y Robayo (2017) identifican los mecanismos por medio de los cuales se implementan las acciones de Orientación al Mercado en las Pequeñas Empresas productoras del sector del calzado en cuero de la ciudad de Bogotá, para los empresarios de este sector los negocios son vistos más desde la perspectiva de la producción y no tanto desde el punto de vista del mercadeo y del análisis del sector.

Por su parte, Sandrea, Boscán y Figuera (2005) indagan el desempeño económico de las PYME zulianas fabricantes de calzado y caracterizan el desempeño comercial, nacional e internacional del recurso humano, así como el proceso productivo y financiero, llegando a la conclusión que el desempeño económico de las empresas estudiadas es deficiente, lo que revela un bajo nivel competitivo en el ámbito regional y nacional al tiempo que no compiten en el ámbito internacional. García (2013) analizó el comportamiento competitivo del 
conjunto empresas productoras de calzado de cuero en Colombia, utilizando los índices de ventaja comparativa revelada, para examinar la dinámica comercial frente al mercado de Estados Unidos y el mercado mundial en distintos tramos del período comprendido entre 1980 a 2008, para poder proyectar las posibilidades de éxito en el marco de la asunción del Tratado de Libre Comercio con Estados Unidos (TLC). Tinto, Luna y Cisneros (2016) desarrollan la teoría de efectos olvidados para el rescate de la imagen comercial de los artesanos del calzado en el cantón Guacaleo provincia del Azuay Ecuador, determinan las acciones y efectos de mejora de la comercialización a través de variables escondidas con la aplicación de la lógica difusa como nueva herramienta innovadora dentro de la gestión empresarial.

Al hablar de factibilidad en la elaboración de calzado de cuero y suela, este hace referencia al recurso económico necesario para crear un emplazamiento y este a su vez ejecutable dentro del mercado, generalmente este término se utiliza dentro de un proyecto de inversión.

\section{Metodología}

La presente investigación, es de tipo descriptivo con enfoque cuantitativo, para lo cual se aplicó cuestionarios, utilizando la técnica de la encuesta, con el propósito de levantar información a los posibles consumidores, con la finalidad de cuantificar la posible demanda existente en el mercado local, de la misma manera y aplicando la técnica similar se obtuvo información de la competencia a nivel de almacenes, con ello se pudo determinar la demanda potencial insatisfecha dentro del mercado de la ciudad de Azogues. Lo explicado se detalla en la siguiente tabla.

Tabla 1 Demanda potencial insatisfecha

\begin{tabular}{|c|c|c|c|c|c|c|c|c|c|c|c|c|c|c|c|}
\hline \multirow{3}{*}{ TIPO DE CALZADO } & \multicolumn{5}{|c|}{ DEMANDA PROYECTADA } & \multicolumn{5}{|c|}{ OFERTA PROYECTADA } & \multicolumn{5}{|c|}{ DEMANDA INSATIFESCHA } \\
\hline & \multicolumn{5}{|c|}{ AÑNOS } & \multicolumn{5}{|c|}{ AÑOS } & \multicolumn{5}{|c|}{ AÑOS } \\
\hline & 2020 & 2021 & 2022 & 2023 & 2024 & 2020 & 2021 & 2022 & 2023 & 2024 & 2020 & 2021 & 2022 & 2023 & 2024 \\
\hline$\overline{\text { BOTI }}$ & 6208 & 6326 & \begin{tabular}{|l|}
6447 \\
\end{tabular} & 6569 & 6694 & 2915 & 2957 & 2992 & 3023 & 3050 & 3294 & 3370 & 3454 & 3546 & 3644 \\
\hline $\mathrm{CON}$ & 94 & 9611 & 9794 & 9980 & 10170 & 4008 & 4065 & T & 4157 & 4194 & 5424 & 5546 & 5680 & 5823 & 5976 \\
\hline MOCASIN & 12835 & 13079 & 13327 & 13580 & 13838 & 2915 & 2957 & 2992 & 3023 & 3050 & 9920 & 10122 & 10335 & 10557 & 10788 \\
\hline TOTAL & 28475 & 29016 & 29567 & 30129 & 30702 & 9837 & 9979 & 10099 & 10203 & 10294 & 18638 & 19037 & 19469 & 19926 & 20407 \\
\hline
\end{tabular}

Fuente: Elaboración propia

Como se puede observar en la tabla 1, la demanda es superior a la oferta, por consiguiente, existe penetración del producto en el mercado, es decir se termina la existencia de una demanda potencial insatisfecha. La demanda se determinó en función de la información 
obtenida de las encuestas y su proyección se lo realizó en función del porcentaje del crecimiento poblacional; en relación a la oferta este se cuantifico de los datos traídos de la competencia, y en base a un histórico de cinco años de los diferentes almacenes de calzado se proyectó por el método de mínimos cuadrados aproximándose a una curva logarítmica.

Baca (2013) afirma: “demanda potencial insatisfecha es la cantidad de bienes o servicios que es probable que el mercado consuma en los años futuros (...)" (p.57). En condiciones aceptables de mercado la diferencia entre la demanda proyectada y la oferta proyectada, representa la demanda insatisfecha, el mismo Baca (2013) sostiene: "Cuando se tiene los datos graficados de oferta-demanda y sus respectivas proyecciones en el tiempo, ya sea con dos o tres variables, la demanda potencial se obtiene con una simple diferencia, año con año" (p.57).

Para cuantificar los ingresos proyectados, es necesario determinar los precios de cada uno de los tipos de calzado a ofertar, para ello en la mima encuesta de oferta se levantó información con relación a este indicador, utilizando el precio promedio de mercado. Baca (2013) asevera: "precio es la cantidad monetaria a la cual los productores están dispuestos a vender y los compradores a comprar un bien o servicio, cuando la oferta y la demanda están en equilibrio" (p.61); la proyección se lo realizó tomando como referencia el porcentaje de inflación de los últimos tres años del país. Según la siguiente tabla.

Tabla 2 Precios y proyección

\begin{tabular}{|c|c|c|c|c|c|c|c|c|c|}
\hline TIPO DE CALZADO & \multicolumn{2}{|r|}{2020} & \multicolumn{2}{|c|}{2021} & \multicolumn{2}{|r|}{2022} & \multicolumn{2}{|r|}{2023} & 2024 \\
\hline BOTIN CORTO & $\$$ & 37,48 & $\$$ & 39,02 & $\$$ & 40,62 & $\$$ & 42,29 & 44,03 \\
\hline CON CORDON & $\$$ & 48,93 & $\$$ & 50,94 & $\$$ & 53,04 & $\$$ & 55,22 & 57,49 \\
\hline MOCASIN & $\$$ & 57,26 & $\$$ & 59,61 & $\$$ & 62,06 & $\$$ & 64,61 & 67,27 \\
\hline TOTAL & $\$$ & 143,67 & $\$$ & 149,58 & $\$$ & 155,72 & $\$$ & 162,12 & $\$ 168,79$ \\
\hline
\end{tabular}

Fuente: Elaboración propia

Demostrar la factibilidad de un nuevo emplazamiento, es un reto para el inversionista, para ello se hace necesario conocer los flujos de caja proyectados para un horizonte de cinco años, estos se derivan de la diferencia entre los ingresos totales menos los costos y gastos que genere el proyecto de inversión, en el último año proyectado se considera el valor de salvamento de los activos fijos y el capital de trabajo, el primero se refiere a la diferencia del valor de adquisición menos el valor depreciado en los cinco años proyectados; en cambio el capital de trabajo es un rubro económico necesario para dar inicio con las actividades del emplazamiento dentro de un corto plazo. Estos flujos de caja, se presenta en la tabla 3. 
Tabla 3 Flujos de Caja Proyectados

\begin{tabular}{ll}
\hline AÑOS PROYECTADOS & FLUJOS DE CAJA NETO \\
\hline 2019 & $-\$ 93.134,85$ \\
2020 & $\$ 14.835,59$ \\
2021 & $\$ 20.104,84$ \\
2022 & $\$ 29.558,58$ \\
2023 & $\$ 40.617,45$ \\
2024 & $\$ 107.873,20$ \\
\hline
\end{tabular}

Fuente: Elaboración propia

A partir de los flujos de caja proyectados, se calcula la tasa interna de rendimiento (TIR) y el valor actual neto (VAN), indicadores de rentabilidad económicos que demuestran la factibilidad del proyecto con dinero a través del tiempo. Para el cálculo de estos dos indicadores, es necesario determinar la inversión necesaria en propiedad, planta y equipo (activos fijos), activos intangibles y capital de trabajo; también se determina la tasa mínima atractiva de rendimiento (TMAR), este porcentaje representa lo mínimo que el proyecto deberá ganar. Sapag (2011) afirma:

El valor actual neto es el método más conocido, mejor y más generalmente aceptado por los evaluadores de proyectos. Mide el excedente resultante después de obtener la rentabilidad deseada o exigida y después de recuperar toda inversión. Para ello, calcula el valor actual de todos los flujos futuros de caja, proyectados a partir del primer periodo de operación, y le resta la inversión total expresada en el momento 0. (p.300)

Las formulas y su aplicación se desarrollan a continuación.

$$
\begin{gathered}
V A N=\frac{F N E 1}{(1+i)^{1}}+\frac{F N E 2}{(1+i)^{2}}+\frac{F N E 3}{(1+i)^{3}}+\frac{F N E 4}{(1+i)^{4}}+\frac{F N E 5+V S}{(1+i)^{5}}-\text { INVERSIÓN } \\
V A N=\frac{14.835,59}{(1+0,17)^{1}}+\frac{20.104,84}{(1+0,17)^{2}}+\frac{29.558,58}{(1+0,17)^{3}}+\frac{40.617,45}{(1+17)^{4}}+\frac{107.873,20}{(1+0,17)^{5}} \\
\quad-93.134,85
\end{gathered}
$$

Desarrollando la formula se tiene. 
$V A N=20.884,42$

Baca (2013) afirma: "La tasa interna de rendimiento (TIR), es la tasa de descuento por la cual el VAN es igual a cero. Es la tasa que iguala la suma de los flujos descontados a la inversión inicial" (p.209).

Para calcular la TIR, se tiene que aplicar el método de interpolación, su aplicación se explica de la siguiente manera.

$$
0=\frac{F N E 1}{(1+i)^{1}}+\frac{F N E 2}{(1+i)^{2}}+\frac{F N E 3}{(1+i)^{3}}+\frac{F N E 4}{(1+i)^{4}}+\frac{F N E 5+V S}{(1+i)^{5}}-\text { INVERSIÓN }
$$

Esto significa que se buscará una tasa ( i ) que iguale la inversión inicial a los flujos netos de caja del proyecto, que es lo mismo que buscar una tasa que haga el VAN igual a cero. El método de interpolación se desarrolla aplicando los siguientes pasos:

a) Se elige la tasa que resulte inferior de las que se examinaron.

b) A esa tasa se le adiciona el producto de la multiplicación del diferencial de la tasa que resulta de la tasa mayor disminuida la tasa menor; y la tasa resultante que multiplica al cociente de dividir el valor presente neto del flujo uno (mayor), entre la suma del valor presente neto del flujo uno (mayor) menos el valor presente neto del flujo dos (menor).

El desarrollo descrito se representa en la siguiente fórmula:

$T I R=$ Tasa menor $+($ Tasa mayor - tasa menor $)\left(\frac{\operatorname{VAN}(+)}{\operatorname{VAN}(+)-\operatorname{VAN}(-)}\right)$

Aplicando:

$T I R=23+(25-23)\left(\frac{4.162,20}{4.162,20+1.280,49}\right)$

Desarrollando la formula se tiene.

$T I R=24,52 \%$ 
Como parte de la metodología presentada en este estudio, se calcula la rentabilidad de la inversión del emplazamiento determinando los indicadores económicos calculados como TIR y el VAN, estos demuestran la factibilidad del proyecto.

El periodo de recuperación de la inversión, está dado por el plazo o tiempo necesario para que los flujos netos de caja, recuperen su costo o inversión inicial. Baca (2013) asevera: “(...) consiste en determinar el número de periodos, generalmente en años, requeridos para recuperar la inversión inicial emitida por medio de los flujos de efectivo futuros que generará el proyecto" (p.212).

Para el caso de estudio, la recuperación de la inversión está dado por los flujos de caja proyectados en los cinco años determinados en la tabla 3, para ello se sigue el siguiente procedimiento:

a) Se suma los flujos de caja, hasta que no sea mayor a la inversión requerida, se considera el año en el que recae este resultado, en este caso tercer año (3).

b) De la inversión estimada, se resta el resultado de la suma de los flujos proyectados. Esta diferencia se divide entre el siguiente año proyectado del flujo de caja. $(0,71)$

c) A este resultado 0,71 se multiplica por 12 , valor que corresponde el número de meses que tiene el año. $(8,46)$

d) Del resultado 8,46, la parte decimal 0,46 se multiplica por 30, valor que corresponde al número de días que tiene el mes. $(13,80)$.

La tabla 4, explica lo descrito.

Tabla 4 Período de recuperación de la inversión

\begin{tabular}{|l|l|l|}
\hline AÑOS & MESES & DIAS \\
\hline $\mathbf{3}$ & $12 * 0,71$ & \\
\hline & $\mathbf{8 , 4 6}$ & \\
\hline & & $30 * 0,46$ \\
\hline \multicolumn{2}{|l|}{} & $\mathbf{1 3 , 8 0}$ \\
\hline \multicolumn{2}{|l}{ 3 años, 8 meses 14 días } \\
\hline
\end{tabular}

Fuente: Elaboración propia

Con relación a este cálculo, se puede decir que la inversión inicial será recuperada dentro del tiempo de 3 años, 8 meses y 14 días aproximadamente. 


\section{Resultados}

Los indicadores económicos de rentabilidad, han demostrado ser los más ideales frente a la tasa mínima atractiva de rendimiento (TMAR), obtener un VAN de 20.8884,42 dólares esto representa una ganancia para el proyecto después de ganar la TMAR. Una TIR del 24,52\% este porcentaje describe la rentabilidad del emplazamiento, ya que es superior a la tasa mínima aceptable de rendimiento, esto significa que este indicador mide el máximo costo que se podría pagar por el capital. Con los flujos netos de caja proyectados, se establece el periodo de recuperación de la inversión, está considerado dentro de los cincos años como horizonte el proyecto, este indicador apoya aún más su factibilidad.

\section{Conclusiones}

- El realizar un estudio de factibilidad para la fabricación de calzado de cuero y suela para caballeros en la ciudad de Azogues-Ecuador, es un reto y al mismo tiempo muy importante ya que se estaría cubriendo una necesidad en el mercado que es servir a la sociedad con un producto de calidad, estableciendo un nicho de mercado exclusivamente para este tipo de bien, al demostrar que sus indicadores de rentabilidad económicos superan los mínimos establecidos, queda justificado su factibilidad, sabiendo que todo proyecto tiene sus riesgos pero estos hay que saber mitigarlos.

- Una vez demostrado que este nuevo emplazamiento es factible desde la concepción de la rentabilidad económica, se deja abierta la opción de ejecutar el mismo y posteriormente su operatividad, con ello se estaría dando solución al problema de investigación, sirviendo a la sociedad azogueña con un producto diferente al de la competencia y al mismo tiempo acrecentando el desarrollo local.

\section{Referencias bibliográficas}

Baca, G. (2013). Evaluación de proyectos (7ma. edición). México: Mc Graw Hill.

Castillo, M., y Cortellese, C. (1988). La pequeña y mediana industria en el desarrollo de América Latina. Revista Cepal (34), 212.

García, J. (1999). ¿Pueden exportar las Pymes?. Revista “Debates IESA”, 1, 50.

García, R., y Maldonado, A. (2013). Competitividad del calzado de cuero colombiano: Perspectiva De la ventaja comparativa revelada (1980-2008). Dimensión 
Empresarial, $\quad 11(1), \quad$ 77-91. $\quad$ Recuperado de http://ojs.uac.edu.co/index.php/dimension-empresarial/article/view/162/146

Mosquera, F., Rojas, S., y Robayo, O. (2017). Orientación al Mercado en las Pequeñas Empresas del Sector Calzado en Cuero de la Ciudad de Bogotá. Investigación Administrativa, 46(120), Recuperado de http://www.redalyc.org/articulo.oa?id=456052444003

Rosales, R. (2000). Estrategias gerenciales para la pequeña y mediana empresa. Ediciones IESA. p 131.

Sandrea, M., Boscán, M., y Figuera, J. (2005). Desempeño económico como factor de competitividad de las PYME zulianas productoras de calzado. Multiciencias, 5(1), 17-29. Recuperado de http://www.redalyc.org/articulo.oa?id=90450103

Sapag, N. (2011). Proyectos de Inversión Formulación y Evaluación (2da. edición). Santiago de Chile: Pearson.

Tinto, J., Luna, K., y Cisneros, D. (2017). Teoría de los efectos olvidados en el rescate de la imagen comercial de los artesanos del calzado en el cantón Gualaceo provincia del Azuay, Ecuador. Visión Gerencial, 1, 24-42. Recuperado de http://www.redalyc.org/articulo.oa?id=465549683003

Villegas, D., Zapata, H. (2007). Competitividad sectorial internacional. Caso: sector del cuero y del calzado. Entramado, 3(1), 24-49. Recuperado de http://www.redalyc.org/articulo.oa?id=265420385004 


\section{PARA CITAR EL ARTÍCULO INDEXADO.}

Espinoza González, J., Luna Altamirano, K., Cantos Ochoa, M., González Astudillo, X., \& Chamba Esparza, V. (2019). Factibilidad en la elaboración de calzado de cuero para caballero: Caso de estudio. Ciencia Digital, 3(2.3), 125-135. https://doi.org/10.33262/cienciadigital.v3i2.3.567

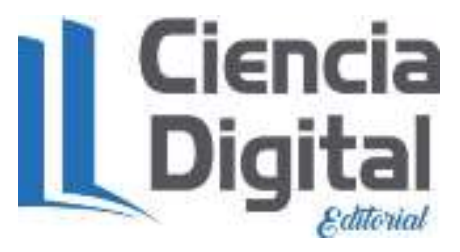

El artículo que se publica es de exclusiva responsabilidad de los autores y no necesariamente reflejan el pensamiento de la Revista Ciencia Digital.

El artículo queda en propiedad de la revista y, por tanto, su publicación parcial y/o total en otro medio tiene que ser autorizado por el director de la Revista Ciencia Digital.
